\title{
¿Más gráficas en los artículos de Cirugía y Cirujanos?
}

\section{More graphs in the papers of Cirugía y Cirujanos?}

\author{
Virgilio Lima-Gómez*
}

Academia Mexicana de Cirugía, Ciudad de México, México

En el área de las finanzas, el análisis empieza por las gráficas; en el ámbito de la educación, realizar una representación gráfica implica habilidades mentales de orden superior, por ser un proceso creativo. ¿Cuál es entonces la razón por la cual se resta relevancia a las gráficas en los artículos de investigación médica?

Los artículos de investigación buscan identificar relaciones entre variables, que pueden expresarse gráficamente; aun cuando solo se reporten frecuencias, una gráfica permite representar medidas de dispersión, que facilitan las comparaciones entre grupos y estimar valores probables de la variable en otras muestras. Cuando las variables evaluadas son continuas, una gráfica ayuda a identificar las características de su distribución, a detectar valores atípicos y a delinear tendencias, que podrían omitirse al revisar los datos en un cuadro.

En los estudios longitudinales, las gráficas pueden mostrar el tamaño del efecto observado sobre las variables, y cuando se elaboran series de tiempo, orientan sobre mediciones de tendencia, estacionalidad, ciclicidad y aleatoriedad. Como estímulo visual aportan otra perspectiva para analizar los resultados de la investigación, y su análisis puede llevar a plantear hipótesis nuevas.

En contra de las gráficas se ha planteado que pueden manipularse, que puede jugarse con la escala para mostrar diferencias inexistentes y otras maniobras que se emplean fuera del ámbito científico para convencer. En la investigación médica esto representa un fraude, que es función de los revisores de una revista identificar; las gráficas, al igual que las conclusiones, requieren sustentarse en los resultados de la investigación, por lo que involucran un ejercicio de síntesis que necesita integrarse adecuadamente.

Algunas escuelas de investigación inician un artículo a partir de una figura, y las gráficas pueden ser la figura que resuma los hallazgos principales de un estudio; los investigadores tienen la oportunidad de seleccionar la representación gráfica que mejor refleje sus resultados, muy probablemente más allá de una imagen que compare $1 \%$ vs. $99 \%$. Como lectores, también podría considerarse profundizar la habilidad para interpretar las gráficas, y evitar la precipitación por omitirlas para llegar a las conclusiones del trabajo.

Como ya se sabe, no todos los trabajos requieren una gráfica, pero elaborarlas es un ejercicio creativo que permite comprender mejor los resultados y comunicarlos de manera más eficiente. El proceso hace posible descubrir opciones de diseño que habitualmente no se exploran, reevaluar lo que se ha concluido y generar una imagen atractiva que cuente la historia de la investigación realizada; una imagen se difunde más fácilmente que un texto y puede despertar el interés para la lectura del reporte completo.

Cada revista tiene sus normas para publicación de figuras, entre ellas las gráficas. Una vez cumplidos esos requisitos, sería una ventaja competitiva

\footnotetext{
Correspondencia:

*Virgilio Lima-Gómez

Avda. Cuauhtémoc 330, 3. ${ }^{\text {er }}$ piso, B

Col. Doctores, Cuauhtémoc

Fecha de recepción: 25-10-2021

Fecha de aceptación: 28-10-2021

E-mail: forscher7@gmail.com

DOI: $10.24875 / C I R U .21000787$

0009-7411/@ 2021 Academia Mexicana de Cirugía. Publicado por Permanyer. Este es un artículo open access bajo la licencia CC BY-NC-ND (http://creativecommons.org/licenses/by-nc-nd/4.0/)
} 
enriquecer las publicaciones con gráficas representativas. Aunque este editorial no tenga una.

\section{Financiamiento}

No se recibió financiamiento para la realización de este trabajo.

\section{Conflicto de intereses}

No existe conflicto de intereses para la publicación de este trabajo.

\section{Responsabilidades éticas}

Protección de personas y animales. El autor declara que para esta investigación no se han realizado experimentos en seres humanos ni en animales.

Confidencialidad de los datos. El autor declara que en este artículo no aparecen datos de pacientes.

Derecho a la privacidad y consentimiento informado. El autor declara que en este artículo no aparecen datos de pacientes. 